REVISTA MATEMÁTICA de la

Universidad Complutense de Madrid

Volumen 10, número 2: 1997

http://dx.doi.org/10.5209/rev_REMA.1997.v10.n2.17455

\title{
Non-maximal cyclic group actions on compact Riemann surfaces.
}

\author{
David SINGERMAN and Paul WATSON
}

\begin{abstract}
We say that a finite group $G$ of automorphisms of a Riemann surface $X$ is non-maximal in genus $g$ if (i) $G$ acts as a group of automorphisms of some compact Riemann surface $X_{g}$ of genus $g$ and (ii), for all such surfaces $X_{g}, \mid$ Aut $X_{g}|>| G \mid$. In this paper we investigate the case where $G$ is a cyclic group $C_{n}$ of order $n$. If $C_{n}$ acts on only finitely many surfaces of genus $g$, then we completely solve the problem of finding all such pairs $(n, g)$.
\end{abstract}

\section{Introduction}

We start with examples of the sort of phenomena in which we are interested. It is known that $C_{7}$ (the cyclic group of order 7) acts as an automorphism group of a Riemann surface of genus 3 . In fact, there are precisely two Riemann surfaces on which $C_{7}$ acts, namely Klein's Riemann surface of genus 3 with 168 automorphisms and a hyperelliptic Riemann surface which necessarily contains an involution in its full automorphism group. (This Riemann surface has $C_{7} \times C_{2} \cong C_{14}$ as its full automorphism group.) Thus even though $C_{7}$ acts as an automorphism group in genus 3 , there is no Riemann surface $X$ of genus 3 with Aut $X \cong C_{7}$. In other words the action of $C_{7}$ in genus 3 is always nonmaximal. This example, though interesting is atypical and in a sense is an accident in low genus. A more typical example is that $C_{12}$ also acts

1991 Mathematics Subject Classifications: 20H10, 30F10.

Servicio Publicaciones Univ. Complutense. Madrid, 1997. 
non-maximally in genus 3 . This result extends to every $g$ in that $C_{4 g}$ always extends to an automorphism group of order $8 g$. However, for each $g$ there is only one compact Riemann surface of genus $g$ that admits a $C_{4 g}$ action. A different situation occurs for $C_{2 g}$ actions. We shall show, with the exceptions of $g=3,6,15$, that $C_{2 g}$ acts on infinitely many Riemann surfaces of genus $g$ and in all cases this action extends to an action of a group of order $4 \mathrm{~g}$.

To be precise we let, for each integer $g>1$,

$$
\begin{aligned}
\mathcal{A}(g)= & \left\{n \in \mathrm{N} \mid C_{n}\right. \text { acts as an automorphism group of some } \\
& \text { Riemann surface of genus } g\}, \\
\mathcal{B}(g)= & \left\{n \in A(g) \mid \text { there is a Riemann surface } X_{g} \text { of genus } g\right. \text { with } \\
& \text { Aut } \left.X_{g} \cong C_{n}\right\}, \\
\mathcal{D}(g)= & \mathcal{A}(g)-\mathcal{B}(g) .
\end{aligned}
$$

Thus $\mathcal{D}(g)$ consists of those values of $n$ such that $C_{n}$ necessarily acts as a non-maximal group of automorphisms of some Riemann surface of genus $g$. The examples above can now be written as $7 \in \mathcal{D}(3), 4 g \in$ $\mathcal{D}(g), 2 g \in \mathcal{D}(g)$ if $g>15$. Another simple example is to take $G$ to be the group of order 1 . Then $G$ is a non-maximal group of automorphisms in genus 2 but not a non-maximal group for genus $g>2$. This is because in genus 2 every Riemann surface is hyperelliptic but for genus $g>2$ there exist Riemann surfaces with trivial automorphism group. (In fact 'most' Riemann surfaces of genus $g>2$ have trivial automorphism group.) Thus $1 \in \mathcal{D}_{(2)}$ but $1 \notin \mathcal{D}(g)$ if $g>2$. The aim of this paper is to investigate the set $\mathcal{D}(g)$. We will see that $\mathcal{D}(g)$ naturally falls into a disjoint union of two subsets depending on whether $C_{n}$ acts on infinitely many surfaces or finitely many surfaces so we let

$$
\begin{aligned}
\mathcal{D}_{0}(g)= & \left\{n \in \mathcal{D}(g) \mid \text { for finitely many Riemann surfaces } X_{g}\right. \\
& \text { of genus } \left.g, C_{n}<\text { Aut } X_{g}\right\}, \\
\mathcal{D}_{\infty}(g)= & \left\{n \in \mathcal{D}(g) \mid \text { for infinitely many Riemann surfaces } X_{g}\right. \\
& \text { of genus } \left.g, C_{n}<\text { Aut } X_{g}\right\},
\end{aligned}
$$

where $<$ here denotes proper inclusion. Thus according to the information in the first paragraph $7 \in \mathcal{D}_{0}(3), 4 g \in \mathcal{D}_{0}(g), 2 g \in \mathcal{D}_{\infty}(g)$ if $g>15$. 
There is no general formula that will determine the integers in $\mathcal{A}(g)$ although Harvey's Theorem(see below) yields an algorithm and for small values of $g$ it is not too difficult to compute $\mathcal{A}(g)$. It is more interesting to determine $\mathcal{B}(g)$ but this is a more difficult problem. We concentrate on investigating $\mathcal{D}(g)$ as it is usually a smaller set than $\mathcal{B}(g)$ and knowing both $\mathcal{A}(g)$ and $\mathcal{D}(g)$ will yield $\mathcal{B}(g)$.

Preliminaries. Let $G$ be a group of automorphisms of a compact Riemann surface $X_{g}$ of genus $g>1$. We shall regard $\left(G, X_{g}\right)$ as a Riemann surface transformation group in the sense of Macbeath $[M]$. We can then lift $\left(G, X_{g}\right)$ to its universal covering transformation group, $(\Gamma, \mathbf{H})$, where $H$ denotes the upper-half complex plane which is the universal covering surface of $X_{g}$ and $\Gamma$ is a cocompact Fuchsian group. Thus there is a homomorphism $\theta: \Gamma \rightarrow G$ whose kernel is the surface group $\Lambda_{g}$ the uniformising group of $X_{g}$. An epimorphism whose kernel is a surface group is called a smooth epimorphism and a necessary and sufficient condition for an epimorphism to be smooth is that it preserves the orders of elements of finite order, see e.g. $[H]$. We shall suppose that $\Gamma$ has signature

$$
\sigma(\Gamma)=\left(h ; m_{1}, \ldots, m_{r}\right)
$$

and presentation

$$
\begin{gathered}
\left\langle a_{1}, b_{1}, \ldots, a_{h}, b_{h}, x_{1}, \ldots, x_{r}\right| x_{1}^{m_{1}}=\ldots x_{r}^{m_{r}} \\
\left.=x_{1} x_{2} \ldots x_{r} \prod_{i=1}^{h} a_{1} b_{1} a_{i}^{-1} b_{i}^{-1}=1\right\rangle .
\end{gathered}
$$

First we recall Harvey's Theorem[H], which gives a necessary and sufficient condition for the existence of a smooth epimorphism froma Fuchsian group to a cyclic group. We use the notation $\left[u_{1}, u_{2}, \ldots, u_{r}\right]$ to denote the least common multiple of $u_{1}, u_{2}, \ldots, u_{r}$.

Harvey's Theorem. Let $\Gamma$ be a Fuchsian group with signature (1.1). Then there exists a smooth epimorphism $\theta: \Gamma \rightarrow C_{N}$ if and only if

(i) $\left.\left[m_{1}, m_{2}, \ldots, m_{r}\right]=\mid m_{1}, \ldots, \hat{m}_{i}, \ldots, m_{r}\right]=M$, where $\hat{m}_{i}$ denotes the ommission of $m_{i}$,

(ii) $M$ divides $N$ and if $g=0$ then $M=N$. 
(iii) $r \neq 1$, and if $g=0$, then $r \geq 3$.

(iv) If $2 \mid M$ then the number of periods divisible by the maximum power of 2 dividing $M$ is even.

We shall call a signature obeying the conditions (i),...(iv) of Harvey's Theorem a Harvey signature.

We shall also be using the Riemann-Hurwitz formula which in our context tells us that if there is a smooth epimorphism from a group of signature (1.1) to a group of order $N$ whose kernel is a surface group of genus $g$ then

$$
2 g-2=N\left(2 h-2+\sum_{i=1}^{\tau}\left(1-\frac{1}{m_{i}}\right)\right) .
$$

Definition. We say that $G$ is a non-maximal group of automorphisms in genus $g$ if (i) $G$ acts as a group of automorphisms of some Riemann surface $X_{g}$ of genus $g$ and (ii) If $G$ acts as a group of automorphisms of a Riemann surface $X_{g}$ of genus $g$ then $\left|A u t X_{g}\right|>|G|$.

Now let $G$ be a non-maximal group in genus $g$, and $X_{g}$ be a surface of genus $g$ on which $G$ acts. If $(\Gamma, \mathrm{H})$ is the universal covering transformation group of $\left(G, X_{g}\right)$ then every Fuchsian group with the same signature as $\Gamma$ is non-maximal and hence must appear in the list of such groups appearing in [S1], where all pairs of signatures $\sigma, \sigma^{\prime}$ are listed with the property that a group $\Gamma$ of signature $\sigma$ is always properly contained in a group $\Gamma^{\prime}$ of signature $\sigma^{\prime}$. In this paper we are only interested in the case when $G \cong C_{n}$ and moreover when we have a smooth epimorphism $\theta: \Gamma \rightarrow C_{n}$ we require it to extend to a smooth epimorphism $\theta^{\prime}: \Gamma \rightarrow G^{\prime}$ where $G^{\prime}$ contains $C_{n}$ with index $\left|\Gamma^{\prime}: \Gamma\right|$. This places further restrictions on $\Gamma$ and also sometimes on the epimorphism $\theta$. Such restrictions were found by Bujalance and Conder in $[\mathrm{BC}]$ and are presented below. To describe their Theorem we will denote the signature $\left(0 ; m_{1}, \ldots, m_{r}\right)$ by $\left(m_{1}, \ldots, m_{r}\right)$; we shall write $\theta\left(x_{i}\right)=z_{i}$ and let $v$ be the generator of $C_{n}$.

Theorem 1. (Bujalance and Conder[BC]). Suppose that $G \cong C_{n}$ acts as a non-maximal group of automorphisms af a Riemann surface $X_{g}$ of genus $g$ and suppose that the universal covering transformation group of $\left(G, X_{g}\right)$ is $(\Gamma, \mathbf{H})$. Then one of the following must hold; 
(a) $\Gamma$ has signature $(n, n, d),(n \geq 3), n+d \geq 7$, d divides $n$ and either $z_{1}=z_{2}$ or $C_{n}$ has an automorphism of order 2 that interchanges $z_{1}$ and $z_{2}$.

(b) $\Gamma$ has signature $(n, n, n)$ and $C_{n}$ has an automorphism of order 3 permuting $z_{1}, z_{2}, z_{3}$ in a 3-cycle.

(c) $\Gamma$ has signature $(3,4,12)$ and $\left\{z_{1}, z_{2}, z_{3}\right\}=\left\{v^{-4}, v^{3}, v\right\}$ or $=\left\{v^{4}, v^{-3}, v^{-1}\right.$

(d) $\Gamma$ has signature $(m, m, n, n)$ where $m+n \geq 5$ and $m$ divides $n$.

(e) $\Gamma$ has signature $(1 ; t, t)$ where $t \geq 2$ and $t$ divides $n$.

(f) $\Gamma$ has signature $(2 ;-)$.

Computation of $\mathcal{D}_{0}(g)$. The distinction between $\mathcal{D}_{0}(g)$ and $\mathcal{D}_{\infty}(g)$ corresponds precisely to whether the universal covering transformation groups $\Gamma$ in the above theorem are triangle groups or not. This is because the dimension of the Teichmuller space of a non-triangle group is positive and hence, for example, there are an uncountanble infinity of groups of signature $(1 ; t, t)$. If we consider a smooth epimorphism from any such group onto $C_{n}$ (where $t \mid n$ ) then each kernel will be a surface group corresponding to some Riemann surface with a non-maximal $C_{n}$ action. On the other hand there is a unique conjugacy class of groups of signature $(l, m, n)$ in $\operatorname{PSL}(2, \mathbf{R})$ and there can only be finitely many epimorphisms of this group onto a triangle group. Hence in order to compute $\mathcal{D}_{0}(g)$ we only need consider cases (a),(b), (c), above.

(a) We let $n=d m$ and suppose $m>1$, (otherwise we are in case (b)). As we want $(d m, d m, d)$ to be a Harvey signature we require $m$ to be even whenever $d$ is even. The triangle group $\Gamma$ has presentation $\left\langle x_{1}, x_{2}, x_{3} \mid x_{1}^{d m}=x_{2}^{d m}=x_{3}^{d}=x_{1} x_{2} x_{3}=1\right\rangle$. We shall suppose that $\theta: \Gamma \rightarrow C_{d m}$ is the smooth epimorphism and that $\theta\left(x_{1}\right)=v, \theta\left(x_{2}\right)=$ $v^{k}, \theta\left(x_{3}\right)=v^{-k-1}$. Now as $v^{k}$ has order $d m$ we have $(k, d m)=1$. As $v^{k+1}$ has order $d, k+1=u m$ where $(u, d)=1$. By Theorem 1 , for $C_{d m}$ to act non-maximally we require that there is an automorphism of order 2 mapping $v$ to $v^{k}$. This implies that $k^{2} \equiv 1 \bmod d m$, or $(u m-1)^{2} \equiv$ $1 \bmod d m$ which is equivalent to the congruence

$$
u m \equiv 2 \bmod d .
$$


We are interested to find when $C_{d m}$ is a non-maximal group in genus $g$. If this holds then for all smooth epimorphisms $\theta: \Gamma \rightarrow C_{d m}$ there is an automorphism of order 2 that interchanges the two generators $v$ and $v^{k}$ of order $d m$. This leads us to consider the following number-theoretic question:

Find all integers $d>1$ with the property that there exists $m>1$ such that for all units $u$ mod $d$,

$m$ is even whenever $\mathrm{d}$ is even and $(u m-1, d)=1$

implies that $u m \equiv 2 \bmod d$.

(The condition $m$ is even whenever $d$ is even or equivalently $(d m, d m, d)$ is a Harvey signature means there is a smooth epimorphism from $(d m, d m, d)$ onto $C_{d m}$ and so there is a unit $u \bmod d$ with $(u m-1, d)=1)$. Once we have found these values of $d$ we search for the corresponding values of $m$. Given such a pair $(d, m)$ we then have a cyclic group $C_{d m}$ with the property that for all epimorphisms $\theta: \Gamma \rightarrow C_{d m}$, there is an automorphism of order two that interchanges the two generators of order $d m$ so that by part (a) of Theorem 1, this cyclic group acts as a non-maximal group on some Riemann surface of genus $g$ where by the Riemann-Hurwitz formula, $g=m(d-1) / 2$. Conversely, if $C_{d m}$ is non-maximal group of some Riemann surface of genus $g=m(d-1) / 2$ and arises from a $(d m, d m, d)$ triangle group (where $m>1)$ then $(d, m)$ obey $(1)$.

Proposition 1. The only integers $d$ that obey (1) above are $d=$ $2,3,4,6,12$.

Proof. We start by finding the prime powers that obey (1). Assume first that $d=p^{\alpha}$ where $p$ is an odd prime. Let $u=1$; then

$$
\left(m-1, p^{\alpha}\right)=1 \text { implies that } m \equiv 2 \bmod p^{\alpha} .
$$

Thus $m \neq k p^{\beta}+1,(1 \leq k<p, 1 \leq \beta<\alpha)$ implies that $m \equiv 2 \bmod p^{\alpha}$. Therefore $m \equiv 2 \bmod p^{\alpha}$ or $m=k p^{\beta}+1$. $(1 \leq k<p, 1 \leq \beta<\alpha)$. We want to show that the second possibility cannot occur. Suppose that 
$m=k p^{\beta}+1 .(1 \leq k<p, 1 \leq \beta<\alpha)$ and let $u=2$ (a unit $\bmod p^{\alpha}$ as $p$ is odd). Then $\left(2 m-1, p^{\alpha}\right)=1$ implies that $2 m \equiv 2 \bmod p^{\alpha}$ and hence

$$
\left(2 k p^{\beta}+1, p^{\alpha}\right)=1 \text { implies that } m \equiv 1 \bmod p^{\alpha}
$$

so putting $m=k p^{\beta}+1,(1 \leq k<p, 1 \leq \beta<\alpha)$ we get $p^{\alpha} \mid k p^{\beta}$, a contradiction. Thus $m \equiv 2 \bmod p^{\alpha}$, and now the statement $\left(2 m-1, p^{\alpha}\right)=1$ implies that $m \equiv 1 \bmod p^{\alpha}$, becomes $\left(3, p^{\alpha}\right)=1$ implies that $2 \equiv 1 \bmod p^{\alpha}$ which is false unless $p=3$. If $\alpha>1$ then the statement $\left(m-1,3^{\alpha}\right)=1$ implies that $m \equiv 2 \bmod 3^{\alpha}$ is false by letting $m=5$ so that the only odd prime power for which (1) can be true is $p=3$, and we easily verify that (1) is true for $p=3$.

We now consider the case $p=2$. We then have to determine the values of $\alpha$ such that $\left(u m-1,2^{\alpha}\right)=1$ implies that $u m \equiv 2 \bmod 2^{\alpha}$. The units $u$ are now all the odd numbers so $\left(u m-1,2^{\alpha}\right)=1$ if and only if $m$ is even, (which also follows from the Harvey conditions). Thus we have to determine when $m$ even implies $m \equiv 2 \bmod 2^{\alpha}$. This fails for $\alpha>2$ by taking $m=4$. Thus $\alpha=1$ or 2 and it is easily seen that $d=2,4$ do obey (1).

We now observe that if $d$ and $e$ are two integers obeying (1) with $(d, e)=$ 1 then de also obeys (1), so that the integers $2,3,4,6,12$ all obey (1). Also if $d$ obeys (1) and $p^{\alpha}$ is a prime power divisor of $d$ then $p^{\alpha}$ obeys (1) so that the only integers obeying (1) are $2,3,4,6,12$.

Having found the values of $d$ it is easy to find the corresponding $m$. (These values must be even). We illustrate this for $d=12$. The units are $u=1,5,7,11$. If $m=2$ then $(2 u-1,12)=1$ gives $u=1$ or 7 and in both cases $u m \equiv 2 \bmod 12$, so $d=12, m=2$ obeys (1). However, $\mathrm{d}=12, \mathrm{~m}=4$, fails as letting $u=5$, we have $(4 u-1,12)=1$ but $4 u \not \equiv 2 \bmod 12$. Similarly $m=5,8$ fail but $m=10, d=12$ also obeys (1), as $(10 u-1,12)=1$ implies that $u=5$ or 11 and in both cases $u m \equiv 2 \bmod 12$. In this way we find all such pairs $(d, m)$ to be as follows:

1. $(2, \dot{m}), m$ even,

2. $(3, m), m \equiv 1$ or $2 \bmod 3$,

3. $(4, m), m \equiv 2 \bmod 4$, 
4. $(6, m), m \equiv 2$ or $4 \bmod 6$,

5. $(12, m), m \equiv 2$ or $10 \bmod 12$.

We sum up in

Proposition 2.The pair $(d, m)$ obeys (1) if and only if $d=2,3,4,6,12$ and $m \equiv \pm 2 \bmod d$.

Proposition 3. The orders of the non-maximal cylic groups of a surface of genus $g$ that arise as smooth images of the triangle groups of signature $(d m, d m, d)(m>1)$ are of all of the form $4 g, 3 g, 8 g / 3,12 g / 5,24 g / 11$.

Proof. The orders of the groups are $d m$ and by the Riemann-Hurwitz formula $g=m(d-1) / 2$. Thus $d m=2 d g /(d-1)$ and letting $d=$ $2,3,4,6,12$ gives the result.

Proposition 3 does not always imply that a smooth cyclic image of $(d m, d m, d)$ is necessarily non-maximal. It is possible that the same cyclic group is a smooth image of a different triangle group giving a maximal action in the same genus. For example there is a non-maximal $C_{12}$ action in genus 4 coming from a smooth epimorphism $(3,12,12) \rightarrow$ $C_{12}$. However there is also a smooth epimorphism $(4,6,12) \rightarrow C_{12}$, and as the measure of the fundamental region of $(3,12,12)$ is the same as that of $(4,6,12)$ it follows from Theorem 1 that there is a maximal action action of $C_{12}$ in genus 4 . It turns out that there are not many of these exceptional cases though to find them all does involve a fair amount of elementary calculations. We indicate the main steps in this calculation. We want to find all signatures $\left(k_{1}, k_{2}, k_{3}\right)$ with the following properties.

1. $\left(k_{1}, k_{2}, k_{3}\right)$ is a Harvey signature with $\left[k_{1}, k_{2}, k_{3}\right]=d m$ where $d=$ $2,3,4,6,12$.

2. The fundamental regions of $\left(k_{1}, k_{2}, k_{3}\right)$ and $(d m, d m, d)$ have the same measure, and thus

$$
1-\frac{1}{k_{1}}-\frac{1}{k_{2}}-\frac{1}{k_{3}}=1-\frac{1}{d}-\frac{2}{d m}
$$

We suppose that $k_{1} \leq k_{2} \leq k_{3}$ and split the calculation into two cases. Firstly, when $k_{3}=d m$ secondly $k_{3}<d m$. In the first case we have

$$
\frac{1}{k_{1}}+\frac{1}{k_{2}}=\frac{1}{d}+\frac{1}{d m}
$$


and thus we see that

$$
d<k_{1}<2 d
$$

and as $\left[k_{1}, k_{2}\right]=d m$ we get

$$
k_{1}+k_{2}-\left(k_{1}, k_{2}\right)=\frac{k_{1} k_{2}}{d}
$$

where $\left(k_{1}, k_{2}\right)$ denotes the greatest common divisor. From (1.6) it is not difficult to find the exceptional signatures. For example, when $d=2,(1.5)$ gives $k_{1}=3$ and (1.6) implies that $k_{2}=4$ and then $d m=[3,4]=12$ and thus $(3,4,12)$ also maps smoothly onto $C_{12}$. The other cases require a bit more calculation but it is not difficult. Writing $\left(k_{1}, k_{2}, k_{3}\right) \sim(d, d m, d m)$ to denote the statement that there is also a smooth epimorphism from $\left(k_{1}, k_{2}, k_{3}\right) \rightarrow C_{d m}$ we find that

1. $(2,12,12) \sim(3,4,12)$,

2. $(3,12,12) \sim(4,6,12)$,

3. $(3,30,30) \sim(5,6,30)$,

4. $(4,24,24) \sim(6,8,24)$,

5. $(4,56,56) \sim(7,8,56)$,

6. $(6,24,24) \sim(8,12,24)$,

7. $(6,60,60) \sim(10,12,60)$,

8. $(6,132,132) \sim(11,12,132)$,

9. $(6,168,168) \sim(8,21,168)$,

10. $(12,120,120) \sim(15,40,120)$,

11. $(12,840,840) \sim(15,56,840)$

12. $(12,120,120) \sim(20,24,120)$

13. $(12,168,168) \sim(21,24,168)$

14. $(12,264,264) \sim(22,24,264)$ 
15. $(12,552,552) \sim(23,24,552)$

We now consider the second case where $k_{3}<d m$, so that $k_{1}<k_{2}<$ $k_{3}<d m$. We still have $k_{1}>d$, for otherwise by (1.4) we have $k_{3}>d m$ contradicting $\left(k_{1}, k_{2}, k_{3}\right)$ being a Harvey signature. Also by (1.4) we have $k_{1}<3 d$. The following result is useful in eliminating many cases.

Lemma. If $k_{1}<k_{2}<k_{3}$ and if $\left(k_{1}, k_{2}, k_{3}\right)$ is a Harvey signature with $\left[k_{1}, k_{2}, k_{3}\right]=N$, then if $k_{1}=p^{r}$ (where $p$ is prime) then $k_{3}=N$.

Proof. We have $\left[p^{r}, k_{i}\right]\left(p^{r}, k_{i}\right)=p^{r} k_{i}$ so that $k_{i}=N\left(p^{r}, k_{i}\right) / p^{r}=N / p^{s_{i}}$, for $i=2,3$. Thus $N=\left[k_{2}, k_{3}\right]=\left[N / p^{s_{2}}, N / p_{3}^{s}\right]$, and so $s_{3}=0$.

It follows that in the second case we can assume that $k_{1}$ is not a prime power. As $k_{1}<3 d$ no possibilities occur when $d=2$ and the only possibility if $d=3$ is $k_{1}=6$. If $(3,3 m, 3 m) \sim\left(6, k_{1}, k_{2}\right)$ then $\left[6, k_{i}\right]\left(6, k_{i}\right)=6 k_{i}$, so that $3 m\left(6, k_{i}\right)=6 k_{i}$ and thus $k_{i}=m / 2$ or $m$, or $3 m / 2$. The only possibility to make $\left(6, k_{1}, k_{2}\right)$ a Harvey signature is that $k_{1}=m, k_{2}=3 m / 2$ and then (1.4) gives $m=6$ and then $\left(6, k_{1}, k_{2}\right)$ is not a Harvey signature. Now suppose that $(4,4 m, 4 m) \sim\left(k_{1}, k_{2}, k_{3}\right)$. As $k_{1}$ is not a prime power and less than $12, k_{1}=6$ or 10 . The same argument as above shows that $k_{2}=4 \mathrm{~m} / 3$ and $k_{3}=2 \mathrm{~m}$ and then (1.4) gives $m=9$ This is impossible by proposition 2 . Similarly we rule out $k_{1}=10$. When we consider the possibilities that $(6,6 \mathrm{~m}, 6 \mathrm{~m}) \sim\left(k_{1}, k_{2}, k_{3}\right)$ we see, as above that $k_{1}=6,10,12,14,15 . k_{1}=6$ or 10 again lead to contradictions. If $(6,6 \mathrm{~m}, 6 \mathrm{~m}) \sim\left(12, k_{2}, k_{3}\right)$, then $\left[12, k_{i}\right]\left(12, k_{i}\right)=12 k_{i}$, so that $k_{i}=\left(12, k_{i}\right) m / 2$. Thus $k_{i}=m / 2, m, 3 m / 2,2 m, 3 m$. As $\left|k_{2}, k_{3}\right|=6 m$, the possibilities are (i) $k_{2}=2 m, k_{3}=3 m$, (ii) $k_{2}=3 m / 2, k_{3}=2 m$. Using (1.4), (i) implies that $m=6$ which contradicts $m \equiv \pm 2 \bmod 6$ in proposition 2. In (ii) we find that $m=10$ which gives the solution $(12,15,20) \sim(6,60,60)$. Similarly, for $d=12$ we obtain a unique solution $(24,30,40) \sim(12,60,60)$.

Thus we can add the final two exceptional signatures as

16. $(6,60,60) \sim(12,15,20)$,

17. $(12,120,120) \sim(24,30,40)$.

\section{Theorem 2.}

For all $g ; 4 g \in \mathcal{D}_{0}(g)$, 
for all $g \equiv \pm 1 \bmod 3,(g \neq 2,4,10) ; 3 g \in \mathcal{D}_{0}(g)$, for all $g \equiv 3 \bmod 6,(g \neq 3,9,21) ; 8 g / 3 \in \mathcal{D}_{0}(g)$, for all $g \equiv \pm 5 \bmod 15,(g \neq 5,10,25,55) ; 12 g / 5 \in \mathcal{D}_{0}(g)$, for all $g \equiv \pm 11 \bmod 66,(g \neq 11,55,77,121,253,385) ; 24 g / 11 \epsilon$ $\mathcal{D}_{0}(g)$.

Proof. The Theorem mostly follows directly from Proposition 2 and the above lists of exceptional signatures. For example $d=12$ gives the orders $24 \mathrm{~g} / 1 \mathrm{l}$, with these occurring precisely when $24 \mathrm{~g} / 11$ is of the form $12 m$ with $m \equiv \pm 2 \bmod 12$. This gives $g \equiv \pm 11 \bmod 66$. The exceptional signatures are those listed as $10,11,12,13,14,15$, and 17 above which give $g \neq 55,77,121,253,385$. There is one other exception, namely $g=11$. This occurs because we will show later in Theorem 4 that $24 \in \mathcal{D}_{\infty}(11)$. Thus $24 \notin \mathcal{D}_{0}(11)$ but we still have $24 \in \mathcal{D}(11)$. The same reason gives the exceptions $2,3,5$ in the second, third and fourth cases. Thus $6 \in \mathcal{D}(2), 8 \in \mathcal{D}(3), 12 \in \mathcal{D}(5)$. In the case when $d=2$, we have $(2,12,12) \sim(3,4,12)$ and by Theorem $1,(3,4,12)$ also gives a non-maximal action of $C_{12}$ in genus 3 , which shows $4 g \in \mathcal{D}_{0}(g)$, for every g. These values in $\mathcal{D}_{0}(g)$ arise out of case (a) of Theorem 1 ; however note that case (c) $(g=3, n=12)$ has already been included in case (a).

We now consider case (b) of Theorem 1 and find the non-maximal groups arising from images of $(n, n, n)$ triangle groups. We note that in order for $(n, n, n)$ to be a Harvey signature $n$ must be odd and as $g=(n-1) / 2$, we can also assume that $n>3$. As before we suppose that the presentation of the triangle group $\Gamma=(n, n, n)$ is $\left\langle x_{1}, x_{2}, x_{3} \mid x_{1}^{n}=x_{2}^{n}=x_{3}^{n}=x_{1} x_{2} x_{3}=1\right\rangle$ and that we have an epimorphism $\theta: \mathrm{\Gamma} \rightarrow C_{n}$ where $C_{n}=\left\langle v \mid v^{n}=1\right\rangle$. We may suppose that $\theta\left(x_{1}\right)=v, \theta\left(x_{2}\right)=v^{k}, \theta\left(x_{3}\right)=v^{-k-1}$. Thus we have the generating triple $\left(v, v^{k}, v^{-k-1}\right)$ of $C_{n}$ and in order for $C_{n}$ to act non-maximally, we need, for all such triples, either an automorphism of order two interchanging two generators of the triple or an automorphism of order three that cyclically permutes them. In order for there to be an automorphism of order two that interchanges two of the generators we need an automorphism $\alpha: C_{n} \rightarrow C_{n}$ with either $\left.\alpha(v)\right)=v^{k}, \alpha(v)=v^{-k-1}$ or $\alpha\left(v^{k}\right)=v^{-k-1}$. That is we need $k^{2} \equiv 1 \bmod n$, or $(k+1)^{2} \equiv 1 \bmod n$ or $k^{2} \equiv(k+1)^{2} \bmod n$. For there to be an automorphism of order 3 we need $k^{2}+k+1 \equiv 0 \bmod n$. If $n$ is a prime then we see that there 
are at most 7 values of $k$ that could satisfy one of these congruences. If $n$ is composite then the total number of solutions is no more that $7^{\nu(n)}$ where $\nu(n)$ is the number of prime factors of $n$. By considering all possible generating triples it is easily seen that for $n=5,7,9,15,21$. such an automorphism must exist. For example when $n=21$ these triples (with $\left.\theta\left(x_{1}\right)=v\right)$ are $\left(v, v, v^{19}\right),\left(v, v^{4}, v^{16}\right),\left(v, v^{10}, v^{10}\right),\left(v, v^{16}, v^{4}\right),\left(v, v^{19}, v\right)$ and in the 1st, 3rd and 5th cases there is an automorphism of order two interchanging the generators and in the 2 nd and 4 th cases there is an automorphism of order three permuting the 3 generators. On the other hand when $n=11$, we have the generating triple $\left(v, v^{2}, v^{8}\right)$ which leads to a maximal action. We now show, with the exceptions noted above, that this will always be the case.

Theorem 3. An epimorphism $\theta: \Gamma \rightarrow C_{n}$ leads to a non-maximal group action if and only if $n=5,7,9,15,21$, corresponding to $g=2,3,4,7,10$ respectively.

Proof. We have already noted that the action is necessarily nonmaximal if $n=5,7,9,15,21$. To prove the converse we need only show that in all other cases the number of generating triples is greater than the number of exceptional values of $k$ where an automorphism necessarily exists. We have seen that the number of such $k$ is bounded above by $7^{\nu(n)}$. The number of values of $k$ giving a generating triple $\left(v, v^{k}, v^{-k-1}\right)$ is the number of pairs of consecutive units $(k, k+1) \bmod n$. By exercise 46 page 74 , section 2.3 of [NZM], this number is

$$
\psi(n)=n \prod_{p \mid n}\left(1-\frac{2}{p}\right) .
$$

(We would like to thank Robert Syddall for pointing out this exercise to us.) Thus we have to compare two multiplicative functions $7^{\nu(n)}$ and $\psi(n)$ and it is enough to do this for prime powers, $p^{t}$. Thus we need all prime powers for which $\psi\left(p^{t}\right)=p^{t}-2 p^{(t-1)}<7$ and this is the case only for $n=3,5,7,9$. Thus all the exceptions have these as prime power factors and it is then not difficult to see that except for $n=3,5,7,9,15,21$ the number of generating triples is greater than the number of exceptions.

Using Theorems 2 and 3 we can find the sets $\mathcal{D}_{0}(g)$ for each $g \geq 2$ precisely. See the final section for examples. 
3. Computation of $\mathcal{D}_{\mathrm{co}}(\mathrm{g})$. As we have already noted at the beginning of section 2 in order to compute the integers in $\mathcal{D}_{\infty}(g)$ we need to consider cases (d),(e),(f) of Theorem 1. For example if $N=[m, n]$, the least common multiple of $m$ and $n$, then there is a smooth epimorphism $\theta: \Gamma \rightarrow C_{N}$. This leads to a non-maximal action of $C_{N}$ on a surface of genus $g=N\left(1-\frac{1}{m}-\frac{1}{n}\right)+1$. To show that $N \in \mathcal{D}_{\infty}(g)$ we need to show that there does not exist another Fuchsian group $\Gamma^{\prime}$, having the same measure of fundamental region as $\Gamma$, whose signature does not appear in Theorem 1 and for which there is a smooth epimorphism $\theta^{\prime}: \Gamma^{\prime} \rightarrow C_{N}$. As an example, let $\Gamma$ have signature $(4,4,4,4)$ and $\Gamma^{\prime}$ have signature $(2,2,2,4,4)$. Both are Harvey signatures and both groups have the same measure of fundamental region. Both groups map smoothly under epimorphisms $\theta, \theta^{\prime}$ onto $C_{4}$ and have as kernel a surface group of genus 3. The kernel of $\theta$ gives a Riemann surface with a nonmaximal $C_{4}$ action. However by choosing a maximal $(2,2,2,4,4)$ group, the kernel of $\theta^{\prime}$ gives a Riemann surface with a maximal $C_{4}$ action and so $4 \notin \mathcal{D}_{\infty}(3)$.

Unlike the case of $\mathcal{D}_{0}(g)$ it is not easy to precisely compute the integers in $\mathcal{D}_{\infty}(g)$. However we will prove

Theorem 4. If $N \in \mathcal{D}_{\infty}(g)$ then

(i) $g-1 \leq N \leq 2 g+2$. Moreover, the lower bound is attained if and only if $(g-1,6)=1$ and $g>6$ while the upper bound is attained if and only if $g=2,3,5,11$.

(ii) If in addition $N \neq 2 g+2$ then $N \leq 2 g$, and this bound is attained if $g \neq 3,6,15$.

Proof.(i) The lower bound follows easily from the Riemann-Hurwitz formula. If the measure of a fundamental region of a group $\Gamma$ is $2 \pi \mu(\Gamma)$ then for the groups of signature (d),(e),(f) in Theorem 1 we have $\mu(\Gamma) \leq$ 2, wheras for a surface group $\Lambda_{g}$ of genus $g, \mu\left(\Lambda_{g}\right)=2 g-2$, so that a non-maximal cyclic group acting on a surface of genus $g$ has order $\mu\left(\Lambda_{g}\right) / \mu(\Gamma) \geq(2 g-2) / 2=g-1$. To see when this bound is attained we first note that if 2 divides $g-1$, then $g-1 \notin \mathcal{D}_{\infty}(g)$. For we can get a maximal $C_{g-1}$ action in genus $g$ via a smooth epimorphism from a group of signature $(1 ; 2,2,2,2)$ onto $C_{g-1}$. If 3 divides $g-1$ then $g-1 \notin \mathcal{D}_{\infty}(g)$. For we can get a maximal $C_{g-1}$ in genus $g$ via a smooth epimorphism 
from a group with signature $(1 ; 3,3,3)$ onto $C_{g-1}$. If $g=6$ then we get a maximal action via a smooth epimorphism from a group of signature $(5,5,5,5,5)$ onto $C_{5}$. Now suppose that $(g-1,6)=1$ and $g>6$. Then a $C_{g-1}$ action in genus $g$ must come from a smooth epimorphism of a group with signature $\left(h ; m_{1}, \ldots, m_{r}\right)$ onto $C_{g-1}$. The Riemann-Hurwitz formula gives

$$
2 h-2+\sum_{i=1}^{r}\left(1-\frac{1}{m_{i}}\right)=2
$$

and as $\left(m_{i}, 6\right)=1$ we have $m_{i} \geq 5$ and an arithmetic calculation gives $h=2, r=0$. By Theorem 1 we must have a non-maximal action.

For the upper bound we note that we can get a non-maximal $C_{2 g+2}$ action in genus $g$ via a smooth epimorphism from $(2,2,2 g+2,2 g+2)$ onto $C_{2 g+2}$. We now consider other signatures giving groups of the same measure which could map smoothly onto $C_{2 g+2}$. By Riemann-Hurwitz such a group $\Gamma$ would have $\mu(\Gamma)=\frac{2 g-2}{2 g+2}<1$. The non-triangle groups $\Gamma$ with Harvey signatues and $\mu(\Gamma)<1$ are easily found and in fact are enumerated in [S2]. Their signatures are $(3,3,3,3), \quad(3,3,4,4),(3,3,5,5),(2,3,3,6),(2,3,4,12),(2,3,5,30)$, $(2,2, m, m)$ and these give $C_{N}$ actions on genus $g$ surfaces where $(g, N)=$ $(2,3),(6,12),(8,15),(3,6),(6,12),(15,30)$ and in the final case $\left(\frac{m}{2}, m\right)$ if $m$ is even and $(m-1,2 m)$ if $m$ is odd. Only in the final case do we get a $C_{2 g+2}$ action in genus $g$. However, there is also a smooth epimorphism from a group of signature $(g+1,2 g+2,2 g+2)$ onto $C_{2 g+2}$ whose kernel has genus $g$. By Proposition 2 this only gives a maximal action if $g=2,3,5,11$. Thus if $g \neq 2,3,5,11, C_{2 g+2}$ is a non-maximal group of automorphisms in genus $g$. For (ii) we note that by the above list of pairs $(g, N)$ that we cannot have $N=2 g+1$. We get a nonmaximal action of $C_{2 g}$ in genus $g$ via a smooth epimorphism from a group of signature $(2,2,2 g, 2 g)$ to $C_{2 g}$. The above list of pairs $(g, N)$ shows that the only case where we get a maximal action is when $\Gamma$ has signature $(2,3,3,6),(2,3,4,12),(2,3,5,30)$ which gives $g=3,6,15$ respectively.

Theorem 4 only partially tells us what the elements of $\mathcal{D}_{\infty}(g)$ are. This is unlike the case of $D_{0}(g)$ where Theorems 2 and 3 give us very precise information. What we find is that if $N$ is close to the bounds $g-1$ and $2 g$ of Theorem 4 then using the arithmetic ideas of the above 
proof we can find out precisely when $N$ belongs to $\mathcal{D}_{\mathrm{oo}}(g)$. Otherwise a great deal of work may be necessary. As an illustration we prove

\section{Theorem 5.}

(i) $2 g-1 \in \mathcal{D}_{\infty}(g)$ if and only if $g=2$ and $g=8$.

(ii) $2 g-2 \in \mathcal{D}_{\infty}(g)$ for all $g \neq 2,3,4,5,6,7,10,11,13,16,22$

(iii) $2 g-3 \in \mathcal{D}_{\infty}(g)$ if and only if $g=4,6,12$.

(iv) $g \in \mathcal{D}_{\infty}(g)$ if and only if $(6, g)=1$.

(v) $g+1 \in \mathcal{D}_{\infty}(g)$ if and only if $(g+1,6)=1$ or $g=2$.

(vi) $g+2 \notin \mathcal{D}_{\infty}(g)$ if $g>2$.

Proof. The proofs are just arithmetic and simple refinements of the proofs in Theorem 4. We just indicate the main points.

(i) The cases $g=2$ and $g=8$ come from smooth images of $(3,3,3,3)$ and $(3,3,5,5)$.

(ii) The reason here is that we can always get a maximal $C_{2 g-2}$ action in genus $g$ by Theorem 1 by mapping a group of signature $(1 ; 2,2)$ onto $C_{2 g-2}$. However we can get non-maximal actions for the exceptional genera listed above. For example, a Harvey signature $\left(m_{1}, m_{2}, m_{3}, m_{4}\right)$ with at least 3 distinct periods and $\sum_{i=1}^{4} \frac{1}{m_{i}}=1$ will give a non-maximal $C_{2 g-2}$ action. These give all the above cases except for $g=2$; for example $g=22$ comes from mapping $(2,3,7,42)$ onto $C_{42}$. We get $g=2$ as an exception from the group with signature $(2,2,2,2,2,2)$ and $g=3$ from $(2,2,2,4,4)$.

(iii) These come from smooth images of $(5,5,5,5),(3,3,9,9),(3,3,7,7)$. (iv),(v) The proofs here are similar to the proof of Theorem 4(i).

(vi) If $g+2 \in \mathcal{D}_{\infty}(g)$ then by Theorem 1 , there must be a smooth epimorphism from a group either with signature $(1, n, n)$ or with signature $(m, m, n, n)$ onto $C_{2 g+2}$ whose kernels are surface groups of genus $g$. By the Riemann-Hurwitz formula we obtain, in the first case, that

$$
3 n=(g+2)
$$


and hence $(g+2)$ is divisible by 3 . However, we can now get a maximal action of $C_{2 g+2}$ in genus $g$ via a smooth epimorphism from a group with signature $\left(\frac{g+2}{3}, g+2, g+2, g+2\right)$ to $C_{2 g+2}$ a contradiction so that $g+2 \notin \mathcal{D}_{\infty}(g)$. In the second case Riemann-Hurwitz gives

$$
\frac{g+2}{m}+\frac{g+2}{n}=3 \text {. }
$$

By Harvey's Theorem, both $m$ and $n$ are divisors of $g+2$. Hence one of the terms in the above sum is 2 and the other is 1 . We can suppose that $\frac{g+2}{m}=2$ and thus $g$ is even. If $g$ is divisible by 4 , then we get a maximal action of $C_{g+2}$ via a smooth epimorphism from a group of signature $\left(2,2, \frac{g+2}{2}, \frac{g+2}{2}, \frac{g+2}{2}\right)$ onto $C_{g+2} ;$ if $g>2$ and $g \equiv 2 \bmod 4$ then we get a maximal action of $C_{g+2}$ via a smooth epimorphism from $\left(2,2, \frac{g+2}{4}, g+2, g+2\right)$ onto $C_{g+2}$. hence for all $g>2$ we can get a maximal action of $C_{g+2}$ in genus $g$.

Examples. Finally we give some examples of the sets $\mathcal{D}(g)$ that can be easily found. Our results should enable us to write down $\mathcal{D}_{0}(g)$ exactly and also the values of $N$ in $\mathcal{D}_{\infty}(g)$ for $N$ close to $g$ or $2 g$. Thus we should be able to compute $\mathcal{D}(g)$ for small $g$, but may need extra work to compute it for larger $g$. We shall write $\mathcal{D}(g)=(\ldots . \ldots)$ where the integers before the bar are those that lie in $\mathcal{D}_{\infty}(g)$ and the integers after the bar are those that lie in $\mathcal{D}_{0}(g)$.

$$
\begin{aligned}
& \mathcal{D}(2)=(3,4,6 \mid 5,8), \\
& \mathcal{D}(3)=(6,8 \mid 7,12), \\
& \mathcal{D}(4)=(5,8 \mid 9,16), \\
& \mathcal{D}(5)=(5,10 \mid 12,15,20), \\
& \mathcal{D}(6)=(7,9,12 \mid 16,24), \\
& \mathcal{D}(7)=(7,14 \mid 21,28), \\
& \mathcal{D}(8)=(7,14,15,16 \mid 24,32),
\end{aligned}
$$

Comments. If $N \in \mathcal{D}(g)$ and $N>2 g+1$ then by Theorem 4, either $N=2 g+2$ or $N \in \mathcal{D}_{0}(g)$, so all these values are determined by Theorems 2,3 and 4. If $N \in \mathcal{D}(g)$ and $N \leq 2 g$ then we have to use Theorem 4 and 5 or, in the cases where these don't apply, (i.e. when $g+3 \leq N \leq 2 g-4$ ) to search for the appropriate smooth epimorphisms in each case or prove 
none can exist. In the above lists this only applies to $g=7, N=10$, and $g=8, N=12$. Here we use smooth epimorphisms from $(3,9,9,9)$ and $(4,4,4,12)$ to show that $10 \notin \mathcal{D}(7)$, and $12 \notin \mathcal{D}(8)$.

The first author would like to thank the staff of the Departamento de Matemáticas Fundamentales at UNED (Madrid) for their hospitality. The ideas of this paper were first worked out during a visit there in the summer of 1996.

\section{References}

[BC] E.Bujalance and M.Conder, On cyclic groups of automorphisms of Riemann surfaces, (preprint, UNED).

[H] W.J.Harvey, Cyclic groups of automorphisms of compact Riemann surfaces, Quart.J.Math. 17(1966),86-97.

[M] A.M.Macbeath, Action of automorphisms of a compact Riemann surface on the first homology group, Bull.London Math.Soc.,5(1973),103-108.

[NLM] I.Niven, H.S.Zuckerman, H.L.Montgomery, An Inroduction to the theory of Numbers, (5th Edition), John Wiley \& Sons 1991.

[S1] D.Singerman, Finitely Maximal Fuchsian Groups, J.London Math. Soc. 6(1972), 29-38.

[S2] D.Singerman, Symmetries of Riemann surfaces with large automorphism group, Math.Ann., 210,(1974), 17-32.

Faculty of Mathematical Studies

University of Southampton

Southampton SO17 1BJ

UK

Recibido: 1 de Julio de 1997

e-mail: dsomaths. soton.ac.uk

Revisado: 22 de Octubre de 1997 